\title{
KARL JASPERS: EL DIÁLOGO COMO ACCESO PROPIO DE LA VERDAD \\ Paula Arizpe*
}

\begin{abstract}
El tema de la comunicación constituye la piedra angular de toda la filosofía de Jaspers; por ello, es necesario definir su alcance. Al mismo tiempo, la comunicación es también la piedra de escándalo, ya que se lleva a cabo dentro del estatuto transobjetivo, pues esta comunicación constituye el puente entre la existencia propiamente dicha y la trascendencia. Al cruzar dicho puente, la existencia encontrará su fundamentación en el ser. En otras palabras, se reconstruirá el camino hacia la unidad de los englobantes divididos que cada existente pleno ha franqueado en su acceso a sí mismo.

La existencia se define, pues, como la orientación al ser qua trascendencia. La íntima conciencia que separa a cada existencia de la trascendencia es individual, y por tanto diferente en y para cada existente, por lo que el proceso comunicativo no arranca desde una plataforma común a todos. Esta aventura comunicativa resulta, por ello, distinta para cada individuo: así como cada uno es medido con respecto a la trascendencia, así cada cual buscará aquello de lo que adolece. Este adolecer resulta doloroso hasta que se completa. Por ello la existencia comunicativa se desarrolla en términos de lucha amorosa.

Así pues, la verdad deviene tal en tanto vivida, y la comunicación es parte constitutiva de la existencia, en tanto recuperación y vivencia.

\footnotetext{
* Departamento Académico de Estudios Generales, ITAM.
} 
El yo, o Existenz de Jaspers, es transobjetivo y se autoconoce solamente en la devoción con que busca su propia realización. El lenguaje de esta comunicación productiva o autocreadora es el silencio, que para él es más elocuente que las palabras mismas. Sin embargo, la precariedad de esta relación podría descorazonar a cualquiera, y por ello implica lucha, y su cura es la infinita voluntad de comunicación, es decir, la voluntad de unidad, de coexistencia, que constituye, en principio, el motor último de toda comunicación. Esta voluntad de unidad está fincada, en última instancia, en la unidad de los englobantes o en la aspiración de la razón a la unidad, en el anhelo de ésta por alcanzar la totalidad dentro del reino de la existencia; en definitiva, la voluntad de unidad está fincada en la trascendencia.

La coexistencia de los existentes plenos no se lleva acabo en el terreno mundano, sino en el ámbito transobjetivo mediante el trabajo del alma en la realización de la existencia en comunidad histórica, en la consolidación de la voluntad absoluta de comunicación. La comunicación es, entonces, el espacio o ámbito que media entre la existencia y la trascendencia, y por ello es el lazo de unión temporal entre los hombres.

74 La comunicación se constituye como tarea infinita, y se fundamenta en la raíz común de la voluntad de unidad. Ésta se dirige de los hombres hacia la verdad que es ser sí mismo. La comunicación no hace más que poner marco a esta necesidad, a este anhelo de unidad, que presupone la fragmentación del ser en distintos englobantes.

La trascendencia como unidad es la garantía última de la comunicación, el espacio en el que toma forma. Por ello, si bien esta unidad es autosuficiente, distante, incomprensible, englobante, no constituye un polo del proceso comunicativo, sino que es propiamente la unidad de todos los polos. La trascendencia como unidad no necesita de la comunicación; ésta pertenece sólo a los hombres, puesto que la comunicación es la búsqueda de aquello que completa; constituye en realidad un defecto, una carencia a superar.

Con esta somera visión de la teoría de la comunicación de Jaspers, podemos detallar la manera como ésta se desarrolla. 
El concepto de comunicación para Jaspers adquiere una connotación muy particular, que debe enmarcarse dentro de la concepción generalizada del término. De acuerdo con el uso común, todo proceso comunicativo tiene dos polos: uno que emite y otro que recibe un mensaje. El proceso que se establece entre estos dos polos se denomina comunicación. En principio, esto parece adecuarse al planteamiento jaspersiano; no obstante, el punto en el cual nuestro autor diverge de la concepción general es en la definición que propone de cada polo, definiciones que habremos de analizar minuciosamente.

La perfectibilidad del individuo cognoscente constituye uno de los polos comunicativos. Esta primera perfectibilidad implica como base una autoaclaración de sí mismo. Dicho autoconocimiento, que tiene como resultado la propia autocreación, constituye todo un proceso de unificación de alteridades dialécticas, es decir, de trabajo sobre sí.

El desarrollo consciente del individuo debe plenificar su existencia, y de ahí llevar a cabo un ambicioso proyecto desde sí que le conduzca a la plenitud. Esto se entiende mejor si analizamos el crecimiento del sujeto en términos existenciales, poniendo especial énfasis en el crecimiento propio del existente concreto como realizador de su propio proyecto de vida. De esta manera, el sujeto puede comunicarse, o hacerse uno, como totalidad constituida y real, con lo que es, fundamentalmente, otro. Este otro, en tanto polo dialéctico alternativo, se entenderá en un primer momento, como mundo; en un segundo momento, como los otros hombres o existentes plenos y, finalmente, como la trascendencia. En definitiva, abordaremos la comunicación en su sentido más amplio, analizando la posibilidad de comunicación desde todas sus facetas. Accederemos a los reductos más íntimos de la personalidad concreta del existente mediante descripciones fenomenológicas un tanto minuciosas, para poder delimitar este proceso comunicativo y lograr entrever de la manera más clara posible el otro término de la comunicación, 'lo otro’ en tanto trascendencia.

El hombre alcanza su verdadero ser en la comunicación, pues no puede acceder a él sin los otros. Yo sólo soy en la comunicación con el otro. Es importante enfatizar este hecho de manera suficiente. La pleni- 
tud de ser-sí-mismo depende de la comunicación con el otro, de ahí la importancia medular del tema en tanto acceso al auténtico ser.

La vida, que mediante la comunicación es existencia plena, puede ser acometida como una empresa personal desde el yo profundo y sus decisiones libres; sólo así la vida será auténtica.

La comunicación surgida de la vivencia en el nivel del yo empírico, será general, superficial, despersonalizada. No es comunicación sino incomunicación, soledad e insatisfacción. Sólo en la instancia del yo profundo surgirá la comunicación existencial, verdadera comunicación de la existencia auténtica.

Dado que esta comunicación existencial es histórica, pues se da dentro de unas coordenadas espacio-temporales determinadas, tiene como cuerpo físico aquella comunicación empírica que, debido a sus características objetivas, puede aparecer y manifestarse dentro del mundo. Sin embargo, la verdadera comunicación, la que pretendemos delimitar en este apartado, pertenece al plano existencial. En otras palabras, la verdadera comunicación es aquélla en la que verdaderamente se esclarece el origen, el cual se conoce en tanto se produce con el otro yo; por ello, su pensamiento es de carácter filosófico. Esto significa que no se trata de un saber llanamente objetivo, sino que va implicada la existencia, en su totalidad, de ambos sujetos inmersos en la relación comunicativa. Sin embargo, para alcanzar la cima de este proceso comunicativo, el hombre ha de unificarse y posteriormente hacerse otro con cada uno de los planos fundantes de su vida, tanto externos como internos. Es decir, el hombre debe unificar los englobantes intra se.

Este planteamiento se aclara si recordamos que, en las conceptualizaciones de Jaspers, el hombre es un ser inacabado en búsqueda de autorrealización constante y, en este sentido, su existencia es un trascender; por ello, el hombre no debe saltarse ningún peldaño. La disposición a una comunicación ilimitada no surge como fruto de un conocimiento, sino de la resolución de ser hombre. Se trata de una decisión; nace a partir de la voluntad absoluta de comunicación, anterior a la razón. Creer en la comunicación es un asunto personal, por lo que nuestro 
autor enmarca la comunicación en el terreno de la fe, como una posibilidad real presente en cada uno de nosotros, pero que tiene que realizarse aún. A través de este camino es como se accede a la verdad existencial, y por consiguiente, llega a serla propiamente en sí mismo, no sólo a pensarla. La mismidad propia y la comunicación están en una interrelación tan estrecha que no se da ninguna mismidad sin comunicación ni comunicación sin auténtica mismidad.

Éste ir de camino tiene un punto de partida, mi ‘existencia’ como existencia empírica; un punto de dirección, la trascendencia; y el camino mismo, que es la comunicación. De ahí que deba subrayarse suficientemente la importancia de la comunicación para llegar a ser sí mismo y alcanzar la trascendencia. La existencia es, pues, historicidad y libertad, incondicionalidad en las situaciones límite, en la conciencia y en la elección. La dialéctica de unidad constituye el hilo conductor de las relaciones de logro y abandono, de la 'ley del día y pasión de la noche' entre la existencia y la trascendencia.

Paralelamente, recordemos que la razón tiene, dentro de esta concepción, un carácter unitivo con respecto a la existencia empírica que separa y concreta los fenómenos a su alcance. La razón constituye, así, el campo de la verdad ilimitada, sin fragmentación, cuando ha trascendido la empiricidad. Por ello, más allá del terreno objetivo, la razón ha de entenderse no ya como anhelo de unificación, sino como voluntad de comunicación que tiende a lo uno o al ser, entendido éste como totalidad absoluta. En este sentido, Jaspers afirma que la razón es una y la misma que el deseo de comunicación. La razón, abierta a todo, se endereza a lo Uno de todo ente, impide que la comunicación se interrumpa y con imperturbable confianza exige siempre aventurar de nuevo la comunicación en las ilimitadas posibilidades desde la totalidad del ser.

A continuación, detallaremos el desarrollo del proceso comunicativo desde sus inicios. Nótese, a lo largo de este análisis, el correr paralelo de los conceptos de existencia y comunicación. 
PAULA ARIZPE

\section{La base: comunicación empírica}

La estructura esencial del hombre es de relacionalidad con el mundo y de coexistencia con los demás hombres. Por tanto es un ‘quehacer', una llamada a la realización y esta posibilidad sólo es dada en la comunicación. La comunicación, entendida como la vida con los otros hombres, se da de infinitas maneras dentro de las relaciones colectivas observables en el seno del mundo empírico, susceptibles de análisis tanto en sus motivos como en sus fines. Sin embargo, estas formas de comunicación que se llevan a cabo dentro del plano empírico no constituyen, ni pálidamente, aquella comunicación existencial cabal en la cual la existencia como tal se realiza con el otro, entendiendo a este otro también como existente pleno.

La comunicación existencial es el único camino válido para desdoblar las posibilidades más profundas y características del ser humano. La verdadera comunicación, en la que sólo verdaderamente conozco mi ser, no existe empíricamente, sino que su aclaración es una tarea filosófica. Al decir yo estamos diciendo a la vez mundo. Lejos de estar el hombre preso en su subjetividad, ésta consiste en estar abierto a las cosas bajo una nueva especie: ni como una cosa del mundo, ni como un yo cerrado en él, sino como vida, como quehacer del yo con las cosas, como existencia definida por su carácter ontológico fundamental de estar en el mundo. La vida es una polémica con el mundo, que llamamos realidad, es lucha, acción, formación, es frustración en él, es adaptación a él, es aprehensión y saber acerca de él.

Por tanto, la comunicación es el llamado absoluto y primigenio de la razón y la libertad que decide autónomamente: el hombre que no trasciende 'hacia', sino que es una radical afirmación de su gestarse, de su continuo sobrepasarse y de su transición, un absoluto despliegue y profundización de sí mismo. El estar abierto a la comunicación es una autoafirmación.

Este recorrido empieza desde la existencia empírica; de ahí van surgiendo obstáculos que es preciso superar paulatinamente para acceder 
a los posteriores estadios de la existencia plena como tal. A partir del punto de la existencia plena, las formas de alteridad o 'ser-con-el-otro' revisten ya la forma de comunicación propiamente, que ya no es algo objetivo, sino esencialmente transobjetivo: constitutivo del ser propio que se identifica con el trascender. Este trascender es solamente posible mediante la realización con otras existencias. De este modo, la unidad y alteridad se traducen en el existir insubstancial como objetividad social y en la existencia plena como mismidad subjetiva. El ámbito de la comunicación verdadera es, pues, el de la auténtica existencialidad.

Los tres modos de ser en los que se diversifica lo englobante tienen una correspondencia de tipo unívoco dentro de los planos comunicativos que plantea Jaspers como acceso al verdadero ser. Veamos existencialmente este primer plano empírico, o elemento constitutivo de la comunicación.

\section{El punto de partida: comunicación primitiva o elemental}

El punto inicial de la aventura humana hacia ser-sí-mismo se da cuando el hombre enfoca su interés egocéntrico en persecución de la conservación y propagación de la vida. Estos instintos sensibles primarios se experimentan por él, dada la naturaleza específica del hombre. Dicho movimiento se traduce en el ansia de riqueza y de poder que caracteriza a esta existencia de tipo empírico. Cuando en este plano surgen intereses coincidentes con los de los otros, se forman comunidades que persiguen los mismos objetivos, condicionando, consecuentemente, todo entorno al fomento de estos únicos intereses particulares. Así, en este plano, la relación se reduce o bien a la sola expresión de estos intereses, o bien a la lucha encarnizada por ellos. Al quedar satisfechas las necesidades que han dado origen a esta comunidad, es decir, el instinto de conservación, el deseo sexual y la voluntad de poder, la relación sencillamente cesa, no se trata, por tanto, de un verdadero nexo, de una verdadera comunicación, sino tan sólo de una satisfacción. 
PAULA ARIZPE

Evidentemente, en esta dimensión vital o sensible no se pone atención a los medios, sino al éxito y a la utilidad, que constituye su limitado ámbito de verdad, por lo que los polos de la relación constituyen solamente medios en orden a los fines particulares de cada quien. La relación objetiva, en su primer y más sencillo nivel, es como una conciencia colectiva. Es decir, en el estado de existencia empírica ingenua, el hombre unido substancialmente a su comunidad no cuestiona, con lo cual su conciencia se identifica con la de la masa general. En este momento, el hombre aún no se pregunta ni por su ser ni por el ser en cuanto tal. La comunicación se instala en el orden primario e ingenuo de las relaciones y transmisiones de los códigos sociales. Su preocupación, en tanto ser empírico, se limita a aquellas exigencias simplemente sensibles, tales como el alimento, el crecimiento y la reproducción. El solo cuestionamiento implicaría una escisión en la cual se captaría conscientemente como un alter superior con respecto al mundo, y constituiría, así, el primer paso para trascenderlo, al aprehenderse a sí-mismo como algo más. En este plano de la existencia, la conciencia primitiva es clara, coincide con la colectividad y su autoconciencia yace bajo un velo. Se trata de una conciencia que se funde en lo común, que no ha llegado a un estado de autoconciencia.

Es evidente que el sujeto no satisface su voluntad de comunicación con la saciedad sensible; por lo tanto, el yo, en este punto, ha de realizar un salto en el momento en que, al ser consciente de sí mismo, se puede oponer a lo otro (entendido como mundo empírico), y de este modo, ir más allá de la sensibilidad para, así, trascender al siguiente plano, que constituye, a su vez, el siguiente polo dialéctico.

\section{El primer peldaño: la comunicación formal o de la conciencia}

Al trascender el hombre el ámbito sensible en el momento en que se hace consciente de su ser como yo (ser-para-sí), accede al plano lógico-formal. En este plano superior de conciencia sus relaciones son de carácter universal, son como una conceptualización de relaciones de 
finalidad y racionalidad objetivas, lo que denominamos conciencia general.

El hombre capta su independencia originaria con respecto al mundo cuando lleva a cabo esta diferenciación formal. Se crea, en este punto, una relación de yoes empíricos desde una perspectiva formalmente inteligente. Es decir, surge una relación comprensiva, en la cual median las cosas como realidades objetivas, y que aparece cuando el hombre da 'un salto al yo', al comenzar a tener conciencia de sí en oposición a lo otro en el mundo empírico de los objetos.

En otras palabras, la relación comunicativa consciente surge a partir de la comprensión de los objetos del mundo. En este nivel de la verdad importa llegar a un acuerdo en función de reglas, conceptos y categorías mentales de validez general, en donde subyacen como fundamento los principios lógicos de identidad y no contradicción, bajo los cuales se puede juzgar la verdad o falsedad de una determinada cuestión, pero sin llegar al compromiso del sí mismo. El incipiente fenómeno sucede en el ámbito de la ratio, del pensamiento lógico. Este tipo de comunicación abraza una exactitud objetiva capaz de fincar finalidades generales y sus medios son asequibles a todos los hombres por igual. Por ello, la comunicación general es un prerrequisito para la interacción social, al igual que para la lógica, la matemática y la ciencia en general.

La preocupación del existente en este plano corresponde a necesidades de tipo social, económico o comunitario básicamente general. Y los polos de esta relación son también sustituibles a discreción de cada individuo, puesto que lo importante son las ideas generales, no los hombres, y por ello es una relación, desde su origen, inexistencial. Esta incipiente comunicación resulta para el sí-mismo una cierta totalidad, pero insatisfactoria, ya que el sujeto como existencia qua talis no es relevante. Por ello, debe irse más allá de esta relación eidética que en términos existenciales resulta insuficiente.

Cabe también la desvirtuación de esta comunicación formal, si se llegase a aprehender al otro yo como cosa, orillándolo a ser medio de los fines propios, al callar motivos e intenciones egoístas y manipuladoras, con lo cual se cerraría la puerta a una verdadera comunicación. La 
PAULA ARIZPE

relación se volvería una lucha de poder y dominio, olvidando el estatuto existencial del otro. Este segundo modo de comunicación se mueve todavía en el plano de la conciencia general. Por medio de la ratio yo no soy, en verdad, yo mismo, pero sin ella no puedo llegar a serlo. La comunicación se aprehende en las cosas que son idénticas para todo el mundo, pero yo trasciendo más allá de ellas al concebir puramente las cosas. Esto se debe a que el hombre no es solamente el yo formal de la inteligencia ni es sólo existencia empírica como vitalidad. El hombre es portador de una sustancia que, o se mantiene en la oscuridad de una comunidad primitiva, o bien se realiza por virtud de una totalidad espiritual, que se hace consciente, pero nunca suficientemente conocida. Esta comunicación, como idea, trasciende la comunidad de la determinación y finalidad clara para la inteligencia, pero es esencialmente distinta del interés egocéntrico del individuo, sujeto a sus oscuras inclinaciones naturales. No se guía por fines definidos fundamentables, sino mediante la inserción de un sentido, en el cual el individuo se encuentra por encima del mundo en el cual se realiza, al entregarse a él.

A nivel de comunicación racional se descubre una copertenencia de estructuras lógicas, pero no así una propiamente dicha comunicación o comunicación yo-yo, en la que el yo-sujeto y el yo-objeto son los extremos de la relación. Al resultar insatisfactoria esta relación de la conciencia universal con su entorno sociológico en términos formales, la voluntad de comunicación impele a trascenderlo, dando lugar al siguiente plano relacional.

\section{El segundo peldaño: comunicación espiritual o de las ideas}

En seguida haremos referencia a un tercer ámbito en el que el hombre puede entrar en relación comunicativa objetivamente comprobable con otro hombre: la dimensión del espíritu. A diferencia del primer tipo de comunicación, que obedece a instintos, y del segundo tipo, que es meramente formal, este tercer tipo es el de una comunicación con contenidos. 
La mutua comprensión de ambas partes se apoya en una participación de ideas y afinidades comunes que constituye su fundamento.

Este tercer tipo de comunicación, espiritual, abarca dentro de sí las dos anteriores fases como preparación para una comprensión común de ideales universales. En otros términos, esta comunicación consiste en preñar de contenidos las ideas para constituirlas en ideales, lo que establece un sentido dentro de la comunidad humana, y permite cierta creación individual al llevar a cabo los ideales encarnados en la propia existencia a lo largo de las situaciones cotidianas de la vida. Sin embargo, esto no logra todavía tocar el fondo sustancial del individuo particular, lo cual es necesario para que se dé una comunicación de existencia a existencia.

Estas tres formas (sensible, formal, espiritual) constituyen el medio objetivo comprobable de aquella forma de comunicación que se propone como medio idóneo de realización existencial. De esta manera, constituyen formas previas y necesarias para la comunicación existencial, la cual no es posible demostrar empírico-racionalmente, ya que ésta sólo puede ser experimentada transobjetivamente por cada individuo, en su propia vida. Por ello, el acercamiento a la comunicación verdadera es factible de manera indirecta mediante la actividad filosófica, cuya finalidad principal es arrojar luz sobre esta cuestión.

En una primera instancia, se establece que el punto de partida de la objetividad es conditio sine qua non del proceso comunicativo; se trata de un paso esencial en el trascender de la existencia empírica para el ascenso al plano lógico, en el cual funciona la praxis comunitaria. Este plano lógico de las ideas, y se caracteriza como aquél dentro del cual se mueven los individuos a partir de concepciones universales, y en el cual se lleva a cabo la comunicación en función de conceptos generales. Sin embargo, este tipo de comunicación constituye solamente un peldaño más en el camino hacia la verdadera comunicación. La vida en la objetividad de la existencia empírica del mundo sólo es realizable por la participación en ideas, pero el individuo conserva una autonomía, la cual puede abrirse paso en esta objetividad y, por tanto, oponerse a ella. La comunicación en las ideas y su realización 
PAULA ARIZPE

por medio de la existencia lleva al hombre a una mayor proximidad con el otro que la inteligencia, la finalidad y la comunidad primitiva.

Recordemos que la comunicación es el medio por el cual trascendemos hacia lo uno, pero este uno es objetivamente inalcanzable puesto que su ambitalidad es transobjetiva. La unidad, que se logra a través de la razón como ejecutora de la absoluta voluntad de comunicación, no es unidad objetiva, sino búsqueda de este uno englobante en el que todo descansa.

El movimiento de la razón, entendida como la voluntad de comunicación, es una síntesis de polos comunicativos, se aquieta en la unidad de ser, hacia la cual nos movemos, y por la cual luchamos. La razón es, así, un camino ascendente, no un sistema terminado. Es un deleite para el espíritu transitar este camino de la propia vida. La incomprensibilidad de la infinitud de este camino origina el deseo más alto de su comprensión, pues se trata de un anhelo inalcanzable que funge como acicate para traspasar cualquier límite; la razón supera toda objetivación que limite este camino o impida la comunicación. De este modo, toda definición encasilla al ser. El verdadero ser debe estar, entonces, más allá de la escisión sujeto-objeto, es decir, más allá del entendimiento.

En este sentido, como crítica a la falsa certeza aportada por la objetividad, Jaspers piensa que el movimiento que trasciende la orientación intramundana permite al yo hacerse consciente de la relatividad y del límite del conocimiento científico, o impositivo, así como demostrar al mismo tiempo que no es la ciencia la que da sentido desde sí misma a la investigación, sino que aquél le es dado desde un plano superior. El saber objetivo no satisface sus exigencias más íntimas de realización plena.

De este modo, estas relaciones entre individuos de tipo sociológico, siguen una dirección ascendente y se encuentran entrelazadas entre sí:

- la colectividad primitiva conduce a

- la finalidad y racionalidad objetivas, que llevan a

- la espiritualidad, determinada por ideas, con contenido 
Cuando el existente ha experimentado como situación límite personal la insatisfacción de vivir la propia vida al capricho de una colectividad informe, desde su racionalidad objetiva percibe que dentro de esta generalidad no brilla su propia riqueza, o no es auténticamente sí mismo, y lo supera desde un ámbito superior, que es el espíritu. Este movimiento entraña un proceso comunicativo que alerta y afina la conciencia del individuo, integrando en la existencia cada elemento nuevo.

En la última fase se da la apertura de la existencia plena a la trascendencia en al acto de realización existencial. La idea tiene el carácter específico de insertar un sentido al actuar del individuo. En el esclarecimiento de la existencia el hombre se experimenta como una esencia que, en virtud de su libertad e individualidad insustituibles, tiene, por encima de los límites del ser empírico, una dimensión trascendente, y esto le otorga un impulso nuevo para la constatación del ser.

En la comunidad general, o de las ideas, el límite lo constituye el sujeto como sí mismo, al captar su alteridad originaria. En la comunicación entre sujetos inteligentes el límite queda establecido por la universalidad de los contenidos ideales, como totalidades funcionales. Y dentro de la comunicación sustancial, el límite lo determina la existencia misma, que si bien en cada una de las formas precedentes de comunicación se patentiza, y por ende se enriquece, no se agota en ninguna, es un alter superior pero enlazado en alguno de sus aspectos a cada una de las anteriores fases.

La existencia total en su originariedad primigenia se encuentra en comunicación por antonomasia; esta comunicación, dada su transobjetividad, no es aprehensible más que de modo fragmentario en cada una de las manifestaciones de estos tipos de comunicación preambulares, ya que es la totalidad del ser la que está implicada en el proceso comunicativo; la existencia como totalidad, no alguna de sus manifestaciones y menos fragmentariamente.

La aclaración de la existencia reporta un crecimiento espiritual del ser humano en cuanto tal. Este despliegue sólo es posible si la libertad subyace como origen, es decir, bajo la posibilidad de que el hombre 
PAULA ARIZPE

sea dueño de sus actos y los domine desde sí. Este procedimiento acusa una ordenación intrasubjetiva (dentro del hombre mismo), de modo tal que lo inferior se ordene a lo superior. La recta comunicación dentro de las potencias humanas supone para el hombre una alteridad dentro de su propia unidad en tensión, un esfuerzo propio (decisión) sin el cual la tensión entre ellas, y por tanto la plenitud humana, quedaría en suspenso. La falta de trabajo sobre sí mismo impide la realización plena de la existencia humana, por lo que la libertad es la condición de posibilidad de una recta comunicación, tanto intrasubjetiva como intersubjetiva.

El hombre ha de realizarse desde una perspectiva de totalidad, en la cual todas y cada una de sus capacidades se encuentren implicadas y den lo suyo en grado óptimo dentro de una comunicación armónica y, de este modo, pueda considerarse un existente pleno. La objetividad de la realidad empírica de la sociedad es la realidad empírica envolvente, en cuanto que es el mundo de los hombres. Pero del mismo modo, también lo es la vivencia subjetiva que funge como sustrato de la posible existencia, por lo que la dialéctica de unidad-alteridad queda sustancialmente establecida. No obstante, esta comunicación carece aún de 86 la identificación del ser consigo mismo, por lo que es necesario apuntar a un más allá de este límite.

\section{La insatisfacción como motor de la comunicación}

Dado que en cada una de las manifestaciones objetivas de los diversos tipos de comunicación recién enumerados (a saber, la relación sensible, formal y espiritual), sólo algún aspecto del ser se ve enriquecido y captado, derivando una satisfacción parcial, específica y particular. Por ello, en tanto totalidad, la comunicación tiene un amplio margen de insatisfacción. Dentro del proceso comunicativo, en lo que respecta al yo no se está comprometiendo más que cierto aspecto de su existencia: la empírica, la totalidad ideal, el carácter, etc., y bajo determinadas dirección y condiciones. Por ello, considerado el existente como 
unidad completa, experimenta insatisfacción, ya que como sustrato de la existencia está el ansia de dar una respuesta desde la totalidad de su ser. La insatisfacción de la existencia propia comienza al experimentarse la insatisfacción de la comunicación. Tal insatisfacción se hace consciente como clara expresión de la existencia plena, ya que las situaciones límite quebrantan constantemente toda totalidad provisionalmente conquistada.

La falta de realización de la necesidad imperiosa de ser-sí-mismo y la insatisfacción del sujeto dentro de la fase inicial del proceso comunicativo, sirve como límite positivo o acicate, para explorar dentro de sí e intentar aclarar la propia existencia, buscando esa satisfacción, lo que a su vez equivale a develar el origen, es decir, desplegar la propia libertad.

La satisfacción adviene como posibilidad de rebasamiento y superación. La aclaración de la existencia comienza al experimentar la insatisfacción de la comunicación. El malestar a superar también es conditio sine qua non de la autoconciencia, y por tanto de la comunicación auténtica. No hay existencia auténtica para quien no haya atravesado la angustia existencial, que nace de la toma de conciencia, de la experiencia de la soledad total y profunda.

Así, la insatisfacción impele a ir más allá. Y la soledad para un hombre es insatisfactoria. De la co-existencia entre los hombres surge la necesidad de otra conciencia, además de la propia, para confrontarse. Es necesario el reconocimiento del yo en el otro para poder autoconocerse y abrir la puerta a una relación comunicativa.

Por todo lo anterior, Jaspers establece la vida, la existencia como un diálogo. Primeramente a nivel ínfimo o empírico dialogamos con el mundo, lo que nos permite cierto autoconocimiento que abre el diálogo interno. Y así sucesivamente hasta llegar a la instancia suprema del mundo que es su posibilidad vista desde la desnudez de mi propia libertad, y entonces tiene lugar la verdadera comunicación en y por la trascendencia, como compromiso radical, como oxígeno para la existencia.

Quizá ya es tiempo de dialogar con el mundo y con nosotros mismos a otro nivel... 
